\title{
A new sensor for laser tomography on ELTs
}

\author{
E. Gendron ${ }^{1, \text { a }}$, M. Brangier ${ }^{1}$, G. Chenegros ${ }^{1}$, F. Vidal ${ }^{1}$, Z. Hubert ${ }^{1}$, G. Rousset ${ }^{1}$, and F. Pouplard ${ }^{1}$ \\ ${ }^{1}$ LESIA, Observatoire de Paris, CNRS, UPMC, Université Paris Diderot, 5, Place Jules Janssen, 92190 \\ Meudon, France
}

\begin{abstract}
We present a new concept for an optical differentiation wavefront sensor, featuring a high number of phase measurements across the pupil, with a linear response versus the phase gradient. We show measurements with this sensor, obtained though turbulence on elongated laser spots.
\end{abstract}

\section{Introduction}

We present in this article a new type of wavefront sensor. It belongs to the class of optical differentiation wavefront sensors (see review papers from Hénault F.[3], and papers from Oti et al.[1] [2]). It allows us to measure the gradient of the phase. This sensor has the advantage of being linear on a wide range, allows a high spatial sampling of the pupil, and uses only 4 pixels per measurement. Those characteristics makes it a promising candidate for open-loop operation with laser guide stars (LGS) on future extremely large telescopes.

A peculiar sensor property is to have a sensitivity independent from the angular size and shape of the object (within a certain range). The so-called 'centroid gain' effect, well known to affect quad-cell devices, is strongly mitigated here and can even be canceled out. As a consequence of this, the noise variance is also independent from the size and shape of the object.

Because of these interesting properties, we primarily intend to propose this sensor for open-loop measurements (MOAO-type instruments) on laser beacons : the uncontrolled variability of their shape (sodium profile distribution, upward laser spot width) raises calibration issues for any shape-sensitive sensor, that can be solved by this sensor. Unfortunately, the price to pay to get these nice properties is a global increase in the noise figure compared to other sensors.

\section{Description}

\subsection{Principle}

Optical differentiation wavefront sensors work by optically Fourier-filtering the pupil image: placing a varying amplitude transmission filter in the focal plane that will transform the phase gradient into intensity variations in the pupil plane. Instead of using a spatially varying transmissive filter, we will manufacture a spatially varying polarization-rotater. A first Wollaston prism splits the incoming beam into 2 linearly polarized beams, with orthogonal directions of polarization. One of the beam will be used to get the gradient of the phase along $x$, the other along $y$. On both beams, we place the central element of our system : a thin plate that makes the polarization to rotate by an angle $\alpha$, that varies continuously across the focal plane from -45 to +45 degrees over a distance $w$, as described on the left of figure $1(\alpha(x)$ is a linear function of the position $x)$. We will explain how this 'plate' works in the following section. Then a second Wollaston prism acts as an analyzer : its axis is rotated by 45 degrees

\footnotetext{
a e-mail: eric.gendron at obspm.fr
} the original work is properly cited. 
from the first one, so that 4 pupil images are formed at the output (a field lens is inserted somewhere, to form pupil images).

On a non-aberrated beam, all the rays cross the same point (the centre) of our variable rotatory plate, and the polarization is rotated by 0 degrees for any point of the pupil. Hence, the amplitude spreads equally on the axes of the second Wollaston prism, and the intensity is the same for the four pupil images. Instead, an aberrated ray will cross the focal plane at a particular location, characterized by a certain amount of polarization rotation. The second Wollaston prism will translate this rotation into a difference of intensity in the pupil planes. As $\alpha$ is a linear function of the position, getting $\alpha$ is like getting the impact of the ray in the focal plane, i.e. the phase gradient.

Adopting a simple ray-tracing approach to describe the behavior of this sensor, calling $I_{A}(u, v)$ and $I_{B}(u, v)$ the intensity at a pupil location $(u, v)$ in a couple of pupil images formed by the second Wollaston prism, we can retrieve the rotation angle $\alpha$ by :

$$
\alpha=\frac{1}{2} \operatorname{Arcsin}\left(\frac{I_{A}-I_{B}}{I_{A}+I_{B}}\right)
$$

As the quantity $\left(I_{A}+I_{B}\right)$ is the total incoming intensity, we see that the sensor is insensitive to scintillation.

\subsection{The spatially variable rotatory plate}

Some optical materials are said to be optically active. In these materials, the plane of a linearly polarized light will rotate as the beam propagates into the medium, proportionally to the travelled thickness. Any optically active material exists either in dextrorotatory (clockwise rotation) or levorotatory (counter-clockwise) type. Monocrystals of quartz are optically active, but the optical activity is usually masked by the linear birefringence, larger than optical activity by an order of magnitude. Optical activity of quartz is only fully revealed when the light propagates parallel to its optical axis, where its linear birefringence vanishes. At $\lambda=589 \mathrm{~nm}$, the rotatory power of quartz is $21.68^{\circ} / \mathrm{mm}(2.08 \mathrm{~mm}$ make $45^{\circ}$ rotation).
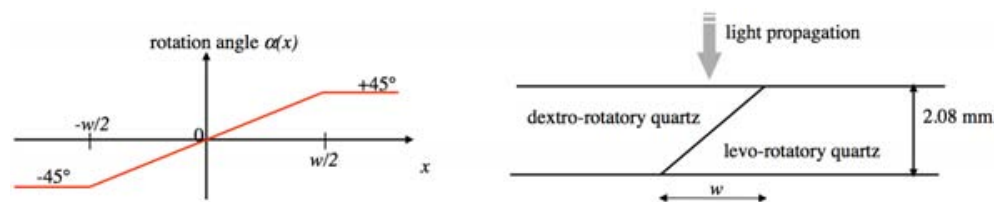

Fig. 1. Left : Variation of the rotation angle of the polarization versus position. Right : Optical design of the spatialy variable rotatory plate. Two plates of a monocrystal of quartz are assembled together. One is dextrogyre, the other laevogyre. The light propagates perpendicular to the plate. The optical axis is parallel to the light propagation. The thickness is $2.08 \mathrm{~mm}$, so that the polarization turns by $+45^{\circ}$ in one crystal, by $-45^{\circ}$ in the other, and of course by any angle in between (as described by the left figure in the zone where the crystals overlap).

We assemble two plates made in a monocrystal of quartz, as described on figure 1 . The width of the wedge (called $w$ on the figure 1) corresponds to the zone into which the sensor will behave linearly. The analyzed beam is to be focused right in the middle of the plate thickness and of the wedge. This particular point defines the center of the wavefront sensor. This is the point where any length travelled in the dextro-plate equals the length in the levo-plate : any rotation will vanish (contrarily to what happens for any other ray). It should be noticed that no particular edge of any material marks the 'zero' of the wavefront sensor (contrarily to to the edge of the knife for Foucault test, or the peak or edges between the faces of a pyramid sensor). Thus, diffractive effects are minimized.

We emphasize that the sensor is not sensitive to the polarization of the incoming light at all, since the first Wollaston prism acts as a polarizer, and thus erases whatsoever previous polarization property. The rotatory power of a material is highly dependent on the wavelength $\lambda$. The dependence is usually 
something close to $\lambda^{-2}$. When used in polychromatic light, one can consider that the sensor will have a sensitivity that varies with $\lambda^{-2}$.
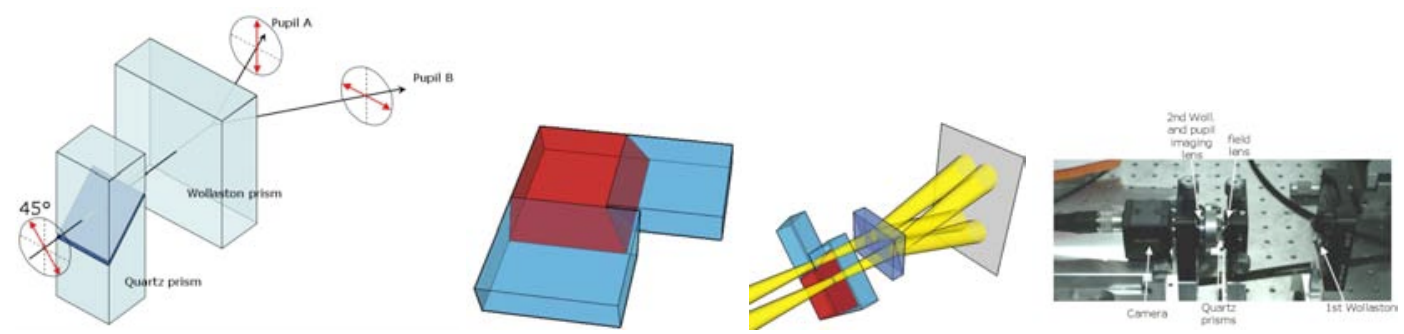

Fig. 2. Left: The polarization rotates proportionally to the height the ray went through the wedge, from $-45^{\circ}$ to $+45^{\circ}$. A Wollaston prism analyzes and splits the beam to form 2 pupil images. Middle left: Picture showing the technical implementation : two orthogonal sides of a quartz plate have been polished with a wedge, and two others plates from the other enantiomer have been cemented to it. Middle right: 3D representation of the setup : light is coming from the left. The two beams have already been separated by a first Wollaston prism. They are both linearly polarized. They are both focused at the center of the quartz wedges. The field lenses have not been represented here. Right: the setup in our lab. Light is coming from the right. The total length from the first Wollaston to the CCD plane is about $100 \mathrm{~mm}$.

\section{Image processing}

Several steps are required in order to get the gradient signal out of the images :

- From equation 1, it is clear that the linearity of the CCD response versus the received flux is of key importance. We have calibrated it, and we have to compensate from a slight non-linearity in the processing.

- The fine subtraction of background is even more important than with a Shack-Hartmann, and the background should be finely calibrated.

- Flat-field also matters a lot, since it impacts directly on the detected photometric signal.

- Due to the non-zero thickness of the quartz plate, the amount of rotation of the polarization has to be corrected from a geometrical effect. One has to apply to the gradient image a kind of "flat-field" correction, which expression (developed at first order in a Taylor series) is : $\left(1+\frac{e}{w} \theta\right)\left(1+\psi^{2} / 2\right)$, with $\theta$ and $\psi$ the angles corresponding to pupil coordinates, respectively parallel and orthogonal to the direction of differentiation.

- Due to aberrations of the optics, the pupil image may reveal some optical distortion (1.5\% in our present design). A special operation -that we call pupil morphing- needs to be performed so that the 4 pupil images accurately match together, pixel-to-pixel. The calibration of this morphing is done beforehand only once, by inserting a square-grid mask in the pupil, and measuring its relative deformation with sub-pixel accuracy in the 4 pupils (correlation).

\section{Experimental results}

We built a prototype sensor, currently tested in our lab within an optical setup that reproduces a f/17 focus of a $4.2 \mathrm{~m}$ telescope. We have chosen a wedge width of $w=1.07 \mathrm{~mm}$ (see fig. 1), equivalent to 2.9 arcsec on-sky. In order to get 4 pupil images arranged on a square, we put the Wollastons' axes orthogonal to each other, and added a quartz plate in between to rotate the polarizations by $45^{\circ}$. The output pupil diameter is $2.2 \mathrm{~mm}$, leading to 228 pixels into the pupil diameter. The sensor is fed by a fiber-source at $\lambda=620 \mathrm{~nm}$, of various diameters. Pupil is at infinity, and may include Kolmogorov-type phase screen. The source can be moved in, $\mathrm{x}, \mathrm{y}, \mathrm{z}$ with precision translation stages. 
The first remarkable thing is that the pupil images are of a very high quality, with no diffracted light outside it (see fig. 3). Rings on the edge of the pupil (derivative of a discontinuous function) do exist, but they are just 2 pixels wide, and not brighter than $\pm 15 \%$ of the average pupil intensity.

We have studied the interaction between optical activity and linear birefringence. We found that a bias is introduced, that is about $0.2 \%$ on the pupil edge for a $\mathrm{f} / 15 \mathrm{beam}$, and behaves as $(f / D)^{-4}$. Although negligible, a solution can be to use a purely optically active crystal, such as $\mathrm{NaClO}_{3}$.

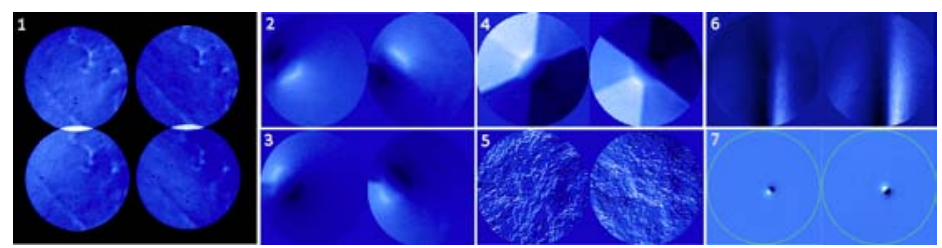

Fig. 3. 1 : Example of raw sensor image, background subtracted only. The patterns inside the pupil are due to inhomogeneity of the incoming flux. Pupil overlap here because the incoming beam is at $f / 15$ (the sensor is designed for f/17). 2 to 7 : Examples of phase gradients after images (flat-field, sub-pixel morphing, combination of images according to eq. 1). The differentiation directions are at $45^{\circ} .2$ and $3:$ phase gradients when applying a voltage on an electrode of a 31-elements bimorph mirror from CILAS. 4 : central electrode of the same bimorph mirror. 5 : SILIOS phase screens manufactured by etching successive binary masks into silica. The very high resolution of the sensor reveals the stair-like structure of the screens (although steps are only $10 \mathrm{~nm}$ high). 6 : Gaz from a cigarette lighter in a 10mm pupil. 7 : one electrode of a $32 \times 32$ electrostatic DM from Boston Micromachines.

\section{Linearity and sensitivity properties}

\subsection{Laboratory tests of linearity on unresolved, extended, and 3D sources}

We have tested the sensor with an unresolved and extended point-sources of 50, 100, 200, and 600 $\mu \mathrm{m}$. The test consists in translating the source by a known amount across the field, and measure the consequent tilt with the sensor in terms of this translation. The experiment was conducted with and without a fixed Kolmogorov-type aberrated plate in the pupil. In any case, we could verify

- a perfectly linear sensor response in terms of the source position

- a slope of this response (the sensor gain) which is independent of the source diameter : no "centroid gain" effect, except on the broadest source $(600 \mu \mathrm{m}$, i.e. 1.62 " wide) that exhibits a relative gain of $\approx 0.90$ compared to the other smaller sources

- no extra centroid gain effect with the presence (or absence) of seeing

We have also simulated an LGS beacon, by defocusing the spot during the exposure time of the camera : we kept the intensity constant during the defocus and this is equivalent to simulate a uniform sodium density profile. We then performed the same linearity tests as before with this defocused source, testing also with different diameters, with or without phase screens. Results are unchanged : the sensor gain is constant, and remains the same for 3D than for 2D sources.

However, in a first time, we have restricted our tests to a limited range of source diameters, defocus, and tilts, in order that the whole blurred image is always still entirely located within the $75 \%$ central part of the wedge. Our wavefront sensor has manufacturing flaws on both sides of the wedge due to aging, and the wedge is not cemented any more at the edge, letting a tiny air gap to appear at both sides, that make a total reflection of light, and that prevents us from using its very whole area.

\subsection{Theory of linearity and gain on 3D extended sources}

A realistic approach that accurately models the sensor for a point source is to use a Fourier-filtering approach : the system forms an image of the pupil by multiplying its Fourier transform (i.e. the com- 

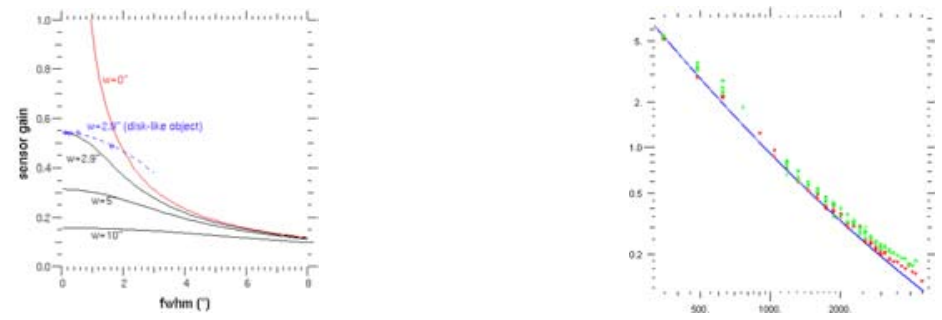

Fig. 4. Left : Theoretical gain of the sensor (arbit. units) in terms of the width of the object (arcsec) for various wedge widths. Plain lines are for gaussian shaped objects, dashed one for a disk-like object. Circles are lab measurements. The red line $\left(w=0^{\prime \prime}\right)$ describes the behavior of quad-cells or non-modulated pyramid. Right : variance of the polarization rotation (in degrees ${ }^{2}$ ) in terms of the flux. The solid line is the theoretical expectation. The different points are for various sizes of object, ranging from point-source to 200 microns wide (equiv. to 0.6 arcsec on-sky). This shows that the experimental noise measurements meet the theoretical expectations.

plex amplitude in the focal plane) by the amplitude-masking functions $m_{x}(x, y)$ and $m_{y}(x, y)$ achieved by the quartz wedge and second Wollaston prism, which expressions are $m_{x}(x, y)=\sin \left(\frac{\pi}{2} \frac{1}{w}(x+w / 2)\right)$ for $x \in[-w / 2, w / 2], m_{y}(x, y)=\sin \left(\frac{\pi}{2} \frac{1}{w}(y+w / 2)\right)$, and replacing $\sin ()$ by $\cos ()$ for the dual functions.

The simulation of an incoherent $3 \mathrm{D}$ extended object is done by considering it as the superposition of incoherent point sources in the field, i.e. by summing the intensities of the filtered pupil for each of these points. This computation quickly becomes too heavy to any computer when the telescope diameter gets larger (the defocus blur grows as $D$ while diffraction is in $D^{-1}$, proportionally leading to $D^{3}$ Fourier transforms of size $D^{4}$ ). We have implemented this algorithm together with speedup computation tricks (as functions $m(x, y)$ only depend on one variable, intensity can be pre-integrated along one dimension of the object ; a point-source shift in the focal plane can be turned into a precomputed shift of the mask $\left.m\left(x-x_{0}, y\right)\right)$ in order to speed up the computation. Despite of this, the computation time remains too long $(\approx$ minutes $)$ to allow its implementation in an end-to-end simulator.

However, the good piece of news is that such a detailed model is useless : thanks to it, we could demonstrate that, as long as the size of the extended object is larger than the diffraction, a simple ray-traced, geometric approach is as valuable as the diffractive one. The intensity at a pupil point $M$ can be obtained by integrating product of $m_{x}^{2}(x, y)$ by the flux of the 3D object as seen from point $M$ : the 2 approaches fit remarkably well. Thanks to this, we could derive the plots shown on figure 4 (left), that show the sensor gain versus the full width at half maximum (fwhm) of the object, assumed to be a gaussian. Those curves exhibit large gain variations because, contrarily to our experiments, the fwhm of the object spans here a much broader variation range (broader than the wedge itself) and that the object has wings (gaussian, instead of the 'disk-like' function of our lab setup). The experimental points we obtained with our lab setup are shown as blue circles on figure 4 (left), on top of the blue dashed line which is the simulation of the sensor gain for a disk-like object.

The red curve has been computed for $w=0$, which corresponds to the case either of a quadcell detector, or a pyramid sensor. This is to compare how this sensor allows us to stabilize the gain, compared to a such a device. In fact, there are 2 regimes : when the object is small compared to $w$ then the gain is stabilized, to a rather fixed value, which is unfortunately lower as $w$ gets larger. On the opposite, when the object is larger than the wedge $w$, the sensor tends to behave as a quad-cell sensor or a non-modulated pyramid : both are hyper-sensitive to centroid gain.

\section{Noise behavior}

We can compute the noise variance on the phase difference between edges of a pixel, assuming that the flux of photons $N$, per frame and per 'pupil sample', is divided equally between the two gradient channels, and then equally divided between the two pupils $A$ and $B$. We assume both photon and detector read-out noise. The noise variance (in $\mathrm{rd}^{2}$ ) is given by: $\sigma_{\phi}^{2}=\left(\frac{2 w_{s k y}}{\lambda / d_{s u b a p}}\right)^{2}\left(\frac{2}{N}+\frac{8 \sigma_{e}^{2}}{N^{2}}\right) \frac{1}{G_{o}^{2}}$ where 
$w_{\text {sky }}$ is the size of the wedge expressed as an angle as seen on the sky, $\sigma_{e}$ is the readout noise in electrons rms per pixel, and $G_{o}$ is the sensor gain (as defined in the previous section) normalized to unity for a point-like object. For any object smaller than the wedge, we have $G_{o}=1$. This expression takes into account the splitting of the flux into 2 channels.

We have experimentally measured the noise variance for small objects $\left(G_{o} \approx 1\right)$ with our setup, by acquiring on a stabilized light source a set of 100 gradients on a fixed wavefront, and computing the time-variance for each pixel (shown vs flux on Fig. 4). The results really show a perfect match with theoretical expectations, and show strictly no dependence of the noise versus source diameter.

Now, the perspective effect makes the apparent size of a laser beacon to vary across the pupil. As the sensor gain depends of the apparent object size seen from a particular pupil position, the sensor gain $G_{o}$ will also vary across the pupil. The gain $G_{o}$ will be equal to $\approx 1$ at any positions where the spot elongation remains smaller than the wedge size $w:$ in these parts, the sensor will be insensitive to any variation of the spot shape. In the other parts, where the elongation exceeds $w, G_{o}$ will degrade to lower values and the noise will increase with spot size just as any other wavefront sensor.

\section{Conclusion}

A new optical differentiation WFS has been described. One of its advantages is to use only 4 pixels per phase measurement, making the detector manufacturing easier for large telescopes : not only a smaller format is required, but also the readout noise spec can be relaxed. The data processing is also lighter and simpler. Another advantage is to provide perfect linearity characteristics, which makes it suitable for open-loop measurements (MOAO) and to provide a stable centroid gain, provided the object is kept smaller than the wedge of the sensor. We see this as a great advantage when dealing with LGSs beacon whose shape may not be under control (upward aberrations, Na profile).

A prototype has been build, and partially tested. Preliminary tests indicate a behavior (noise, linearity, gain) that fits theory remarkably well. In the next months it will be tested with a ELT-type LGS larger than the wedge, to compare the lab performance to theory in these 'extreme' conditions.

The sensor provides a higher noise level than quad-cell, but with the advantage of no 'centroid gain' problem : the balance between those two parameters is tuned by the only free parameter in the sensor design : the wedge size, $w$. A larger $w$ provides increased gain stability and linearity with higher noise, while a small $w$ reduces the range where gain stability and linearity are kept with better noise performance. A $w=0$ reduces the sensor to a pure quad-cell or non-modulated pyramid.

An achromatic version of it has also been developped, that will be presented in future publications.

Acknowledgments Part of this work was funded by the European FP7 Preparatory activity WP 9000 program.

\section{References}

1. Oti J.E., Canales V.F., Cagigal M.P., "Improvements on the optical differentiation wavefront sensor", MNRAS 360, 1448-1454 (2005)

2. Oti J.E., Canales V.F., Cagigal M.P., "Analysis of the signal-to-noise ratio in the optical differentiation wavefront sensor", Optics Express, vol. 11, Issue 21, p.2783, (2003)

3. Hénault, F. "Wavefront sensor based on varying transmission filters: theory and expected performance", Journal of Modern Optics, vol. 52, Issue 14, p.1917-1931, (2005) 